\title{
Introduction to the Special Series: Current Directions for Measuring Parenting Constructs to Inform Prevention Science
}

\author{
Oliver Lindhiem ${ }^{1}$ - Anne Shaffer ${ }^{2}$
}

Published online: 10 November 2016

(C) Society for Prevention Research 2016

\begin{abstract}
Parenting behaviors are multifaceted and dynamic and therefore challenging to quantify. Measurement methods have critical implications for study results, particularly for prevention trials designed to modify parenting behaviors. Although multiple approaches can complement one another and contribute to a more complete understanding of prevention trials, the assumptions and implications of each approach are not always clearly addressed. Greater attention to the measurement of complex constructs such as parenting is needed to advance the field of prevention science. This series examines the challenges of measuring changes in parenting behaviors in the context of prevention trials. All manuscripts in the special series address measurement issues and make practical recommendations for prevention researchers. Manuscripts in this special series include (1) empirical studies that demonstrate novel measurement approaches, (2) re-analyses of prevention trial outcome data directly comparing and contrasting two or more methods, and (3) a statistical primer and practical guide to analyzing proportion data.
\end{abstract}

Parenting is one of the most complex sets of human behaviors, in most instances spanning a minimum of 18 years. During these years, parenting is multifaceted and dynamic and includes the provision of shelter and protection, nurturance, discipline, motivation, teaching, emotional support, instilling values, and passing on traditions. This list is certainly not

Oliver Lindhiem

lindhiemoj@upmc.edu

1 Department of Psychiatry, University of Pittsburgh School of Medicine, Pittsburgh, PA, USA

2 Department of Psychology, University of Georgia, Athens, GA, USA exhaustive, but serves to highlight the vast array of decisions faced by any researcher attempting to quantify the various dimensions of parenting. Prevention researchers who intervene at the level of the family tend to focus specifically the domains of parenting that are associated with undesirable outcomes for children. Although there are many such outcomes that are the focus of prevention (e.g., school drop-out, homelessness, incarceration), all of the papers in this special series specifically address the domains of parenting that are empirically known to be associated with childhood externalizing behavior. Each paper carefully considers one or more methods or measures used to assess parenting, with a range of findings demonstrating the importance of identifying the methods most appropriate to the research questions at hand.

The challenge of operationally defining parenting is, in part, a developmental issue. First and foremost, the measurement of parenting behaviors must be developmentally appropriate. A construct such as "positive discipline" may include time out and redirection in young childhood, but loss of privileges at older child ages. Arguably, the use of time out would be developmentally inappropriate in late adolescence. For example, a parent who attempted to use time out with an older adolescent would be considered to be engaging in ineffective discipline. Longitudinal research and developmental theory have contributed strongly to our sharpened understanding of these issues regarding how positive and negative parenting behaviors can be conceptualized over time.

In addition to the complexities introduced by dynamic changes in parenting over time, measuring and quantifying parenting is challenging for additional practical reasons, including contextual factors and limits on observable behavior. Because the vast majority of parenting behaviors occur in the context of the home, many measures of parenting rely on selfreport or structured interviews. Less frequently, direct observations of parenting are conducted in either the home or lab 
settings. It is not at all surprising that there is no gold-standard measure of parenting, as every approach has disadvantages. A comprehensive review from the prior decade identified 136 different measurement systems (76 questionnaires; 27 interview schedules; 33 observational systems) designed to assess parental discipline and/or nurturance (Locke and Prinz 2002). Questionnaires included parental self-report measures, childreport measures, or both. Interviews included in-person structured interviews, telephone-based structured interviews, and vignette-based interviews. Direct observation systems included frequency and rating methods as well as micro-analytic interactional systems. Problems associated with each approach include social desirability effects and recall biases inherent in self-report forms, the artificial nature of laboratory observations, and the reactivity of home observations (Locke and Prinz 2002). A more recent review identified 164 different measures of parenting published between 1985 and 2009 (Hurley et al. 2014). Although many of these measures lacked strong psychometric properties, there remains a wide enough range of reliable and established measures of parenting that researchers are left with challenging decisions when selecting assessment tools most appropriate to their study goals.

\section{Motivation for this Series}

This series was motivated by recent reports that the specific methods used for assessing and quantifying parenting behaviors have critical implications for study results, particularly for prevention trials that target parenting (e.g., Lindhiem et al. 2014). Although multiple approaches can complement one another and contribute to a more complete understanding of prevention trials, the assumptions and implications of each approach are not always clearly addressed. Greater attention to the measurement of complex constructs such as parenting is needed to advance the field of prevention science. For example, while direct observations of parenting behavior are often considered desirable, relatively little research has directly compared multiple observational tasks or measurement strategies within the same studies or as predictors of the same outcomes. The study of parenting and relations to child behavior has also motivated and benefitted from statistical advances, including longitudinal methods and strategies for understanding dynamic changes over longer and shorter spans of time.

This series examines the challenges of measuring changes in parenting behaviors in the context of prevention trials. All manuscripts in the special series address measurement issues and make practical recommendations for prevention researchers. Manuscripts in this special series include (1) empirical studies that demonstrate novel measurement approaches, (2) re-analyses of prevention trial outcome data directly comparing and contrasting two or more methods, and (3) a statistical primer and practical guide to analyzing proportion data.
The manuscripts in this series include a variety of methodological approaches, including parental self-report (four papers), a semi-structured interview (one paper), and direct observation (two papers). While the papers together represent a range of methods, we have also intentionally included papers that embed comparisons among types of parenting behavior (e.g., "positive" and "negative") and types of measurement techniques (e.g., micro- vs. macro-observational codes, absolute vs. relative frequencies of discipline tactics).

The first paper by Fleming et al. (2016) explores the use of observational measures of child-directed play, parent-directed play, and parent-directed chore tasks as predictors of child conduct problems, with follow-ups to 6 years later. While "analog" behavioral tasks such as these have long been used in the context of clinical and developmental research as a method for obtaining more objective measures of parenting behavior, relatively little is known about their ability to predict child conduct problems over many years. As observational measures are typically more time and labor intensive than self-report measures, such investigations of predictive validity can help shape future assessment planning. Comparisons among the various in-home structured observations showed that parent behavior observed in the child-directed play task were relatively stronger predictors of later child conduct. In particular, the authors report that parental negative attention during child-directed play was especially predictive of both parent- and teacher-reported child conduct problems over 6 years, emphasizing that task-incongruent parental behavioral may be an important risk factor for later problems, and that relatively brief assessments (3- to 5-min tasks) can yield potent longitudinal predictors.

Dishion et al. (2016) address another related issue in the validity of observational assessment of parenting behavior, by directly comparing methods of quantifying observations. Quantitative scales developed for the measurement of observed behavior are most commonly categorized as microlevel, including count/frequency data or moment-to-moment behavioral coding, or macro-level, involving summary scores or global ratings of dimensions of parenting behavior across entire tasks or interactions. In this paper, Dishion and colleagues present a novel coding system designed to capture micro-level parent-child interaction dynamics using statespace grid procedures. They then compare the longitudinal stability and relative predictive utility of this micro-level coding with an existing macro-level coding system designed to capture positive and coercive parenting behaviors over 3 years in families engaged in a preventive intervention, the Family Check-Up. In their analyses, the authors did not find psychometric support for macro-level behaviors as distinguishable latent variables, consistent with concerns that such ratings may be prone to mono-method bias. In contrast, micro-level behavioral measures showed treatment-related improvements in the context of a randomized controlled trial. This paper 
provides discussion of methodological and statistical implications for utilizing micro-codes over macro, including the coding of parent and child behavior separately, operationalization of parent-child interaction dynamics, and consideration of potential coder bias and how to mitigate this possibility.

The third paper by Zheng et al. (2016) describes a personoriented approach to measuring parenting, intended to capture the multidimensional and dynamic nature of parenting. The authors describe the use of growth mixture modeling to characterize patterns of harsh discipline and parental warmth over time. Specifically, they examine how patterns of harsh discipline and parental warmth influence the emergence of child externalizing problems and callous-unemotional traits over time. Their findings highlight the importance of considering the interplay between more than one construct (in this case, harsh discipline and parental warmth) rather than solely investigating these behaviors as independent predictors of child behavior problems.

O'Dor et al. (2016) describe a revised version of the Family Socialization Interview (FSI-R), a comprehensive assessment tool for measuring parental disciplinary behaviors. The FSI-R is a semi-structured interview that assesses both general parenting style and specific discipline techniques (both physical and psychological) over multiple time periods ("past year" and "historical"). The interview also taps into well-being and perceived support of the mother. Of particular relevance for this special series, discipline practices are quantified in more than one way. Specifically, the coding of the interview distinguishes between a typical discipline strategies (termed "usual" discipline) and (termed "most intense" discipline) which are scored separately. The authors present data on the incremental predictive validity for the FSI-R over and above that provided by other self-report and observational measures of parenting, particularly in predicting child internalizing problems.

Shaffer et al. (2016) directly compare two methods for quantifying parenting practices (absolute frequencies vs. relative frequencies or proportion scores). They examine patterns of outcomes using these two methods in the context of a clinical trial to treat and prevent behavioral health problems in primary-care settings using a collaborative care model (Kolko et al. 2014). The paper replicates and extends findings from a prior study (Lindhiem et al. 2014) that measuring discipline practices as absolute frequencies can lead to counter-intuitive results. Specifically, positive parenting techniques (e.g., praise, positive reinforcement) go down over time, rather than up (as might be expected), when quantified as absolute frequencies. When quantified as proportion scores, however, positive parenting techniques increase following intervention. The authors suggest that proportion scores may be a more appropriate way to quantify some constructs related to parenting, particularly in the context of prevention and intervention trials.
Chen et al. (2016) provide a statistical primer to accompany the paper by Shaffer et al. (2016). Specifically, the authors provide guidelines and practical recommendations for performing statistical analyses on proportion scores when they do not conform to statistical assumptions underlying the planned analyses. These assumptions include linearity, normality, and homogeneity of errors, which can be particularly problematic for data points that are close to the boundaries of proportion scores (close to 0.0 and 1.0). Various transformation methods, along with their pros and cons, are discussed. Additional topics include generalized linear models, longitudinal proportional data, and beta regression. Finally, these six articles are followed by an insightful commentary by Eddy (Commentary on the special series: Current directions for measuring parenting constructs to inform prevention science, this issue).

\section{Summary}

This collection of manuscripts brings together experts in parenting interventions, methodologists, and statisticians to provide thoughtful recommendations for measuring parenting constructs in the context of prevention trials. We are hopeful that this collective appraisal of the advantages and disadvantages of various methods and assessment approaches will help guide researchers in selecting the most appropriate measures or statistical techniques for their research questions, which typically focus on parents as "mediators" of preventive intervention content on child behavior outcomes. Measurement is a critically important endeavor in this age in which the replicability and robustness of social science findings is under close examination. We appreciate the opportunity to delve deeply into this issue in a concerted effort across multiple research groups.

In addition, we anticipate that these papers will raise as many questions as they resolve, and thus potentiate new avenues of research for prevention science in the context of families and parenting. Our field needs more research on predictors of parenting behavior in its own right, including the effects of trauma, extreme poverty, and drug addiction. Other important factors that are often neglected included incarceration, immigration, and war (Eddy, Commentary on the special series: Current directions for measuring parenting constructs to inform prevention science, this issue). As noted above, parenting behavior is typically measured and conceptualized as the predictor or mediator in models focusing on child behavior as the outcome. However, as prevention scientists are well aware, individual differences are a very real issue in prevention and intervention research, and consideration of "what works for whom" should also consider contextual factors that predict parenting behavior, including parents' own psychological adjustment, self-regulation abilities, and motivation for treatment (e.g., Chaffin et al. 2011; Maliken and Katz 2013; Sanders and Mazzucchelli 2013). Behavioral parenting 
interventions revolutionized the evidence-based treatment and prevention of child behavior disorders by making parents the delivery agents of the intervention (Forgatch and Patterson 2010), implicating parenting behavior as an important driver of child behavior. Our contemporary developmental and clinical research in this domain draws our attention to the ways in which parents' behavior is influenced by their own context, including the behavior of their children. We believe that future research in this area must incorporate consideration of parenting as the dependent variable, and these considerations of measures that are reliable, valid, and sensitive to change can lead the way.

\section{Compliance with Ethical Standards}

Funding This manuscript was supported in part by a grant from the National Institute of Mental Health (MH093508).

Conflict of Interest The authors declare that they have no conflict of interest.

Ethical Approval This article does not contain any studies with human participants or animals performed by any of the authors.

Informed Consent Because this article is a commentary, informed consent is not applicable.

\section{References}

Chaffin, M., Funderburk, B., Bard, D., Valle, L. A., \& Gurwitch, R. (2011). A combined motivation and parent-child interaction therapy package reduces child welfare recidivism in a randomized dismantling field trial. Journal of Consulting and Clinical Psychology, 79, 84-95. doi:10.1037/a0021227.

Chen, K., Cheng, Y., Berkout, O., \& Lindhiem, O. (2016). Analyzing proportion scores as outcomes for prevention trials: A statistical primer. Prevention Science. doi:10.1007/s11121-016-0643-6c.

Dishion, T., J., Mun, C., J., Tein, J., Kim, H., Shaw, D. S., Gardner, F., Peterson, J. (2016). The validation of macro and micro observations of parent-child dynamics using the relationship affect coding system in early childhood. Prevention Science.

Fleming, A. P., McMahon, R. J., \& King, K. M. (2016). Structured parent-child observations predict development of conduct problems: The importance of parental negative attention in child-directed play. Prevention Science. doi:10.1007/s11121-016-0672-1.

Forgatch, M. S., \& Patterson, G. R. (2010). Parent management training - Oregon model: An intervention for antisocial behavior in children and adolescents. In J. R. Weisz \& A. E. Kazdin (Eds.), Evidence-based psychotherapies for children and adolescents (pp. 159-178). New York: Guilford.

Hurley, K. D., Huscroft-D'Angelo, J., Trout, A., Griffith, A., \& Epstein, M. (2014). Assessing parenting skills and attitudes: A review of the psychometrics of parenting measures. Journal of Child and Family Studies, 23, 812-823. doi:10.1007/s10826-013-9733-2.

Kolko, D. J., Campo, J., Kilbourne, A. M., Hart, J., Sakolsky, D., \& Wisniewski, S. (2014). Collaborative care outcomes for pediatric behavioral health problems: A cluster randomized trial. Pediatrics, 133, e981-e992. doi:10.1542/peds.2013-2516.

Lindhiem, O., Shaffer, A., \& Kolko, D. J. (2014). Quantifying discipline practices using absolute vs. relative frequencies: Clinical and research implications for child welfare. Journal of Interpersonal Violence, 29, 66-81. doi:10.1177/0886260513504650.

Locke, L. M., \& Prinz, R. J. (2002). Measurement of parental discipline and nurturance. Clinical Psychology Review, 22, 895-929.

Maliken, A. C., \& Katz, L. F. (2013). Exploring the impact of parental psychopathology and emotion regulation on evidence-based parenting interventions: A transdiagnostic approach to improving treatment effectiveness. Clinical Child and Family Psychology Review, 16, 173-186. doi:10.1007/s10567-013-0132-4.

O’Dor, S. L., Grasso, D. J., Forbes, D., Bates, J. E., McCarthy, K. J., Wakschlag, L. S. \& Briggs Gowan, M. J. (2016). The Family Socialization Interview-Revised (FSI-R): A comprehensive assessment of parental disciplinary behaviors. Prevention Science.

Sanders, M. R., \& Mazzucchelli, T. G. (2013). The promotion of selfregulation through parenting interventions. Clinical Child and Family Psychology Review, 16, 1-17. doi:10.1007/s10567-0130129-z.

Shaffer, A., Lindhiem, O., \& Kolko, D. J. (2016). Treatment effects of a primary-care intervention on parenting behaviors: Sometimes it's relative. Prevention Science.

Zheng, Y., Pasalich, D. S., Oberth, C., McMahon, R. J., \& Pinderhughes, E. E. (2016). Capturing parenting as a multidimensional and dynamic construct with a person-oriented approach. Prevention Science. doi:10.1007/s11121-016-0665-0. 